\title{
A method for applying haemolysates on starch blocks for electrophoresis
}

P. BARKHAN and M. E. STEVENSON From the M.R.C. Experimental Haematology Unit and the Department of Haematology, Wright-Fleming Institute, St. Mary's Hospital Medical School, London

Electrophoresis of haemolysates on a starch block, which was first described by Kunkel and Wallenius (1955), provides an excellent method for separating and quantitating the various normal and abnormal haemoglobins. Since relatively large amounts of haemoglobin solutions can be applied it is a useful preparative method for isolating a particular haemoglobin for further analysis. One of the important requirements for good electrophoretic resolution on the block is the application of the sample in a narrow band and a simple method for applying the haemolysate is described.

\section{METHOD}

The block is prepared from maize starch (British Drug

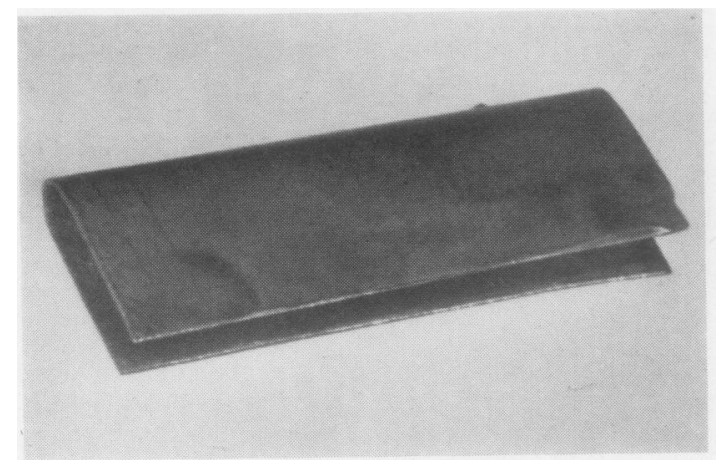

FIG. 1. The U-shaped piece of metal used for making the ridges on the starch block.

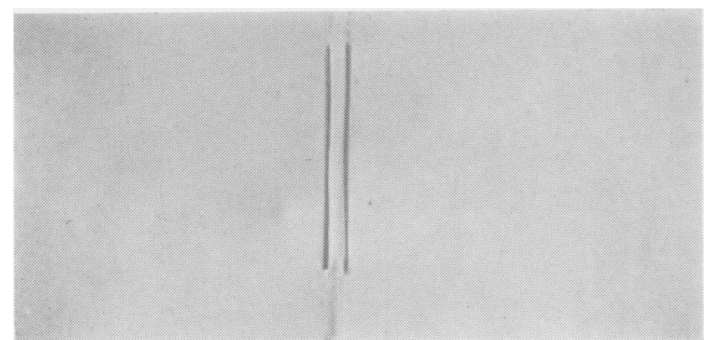

FIG. 2. The ridge before application of the haemoglobin sample.

Received for publication 20 February 1963.

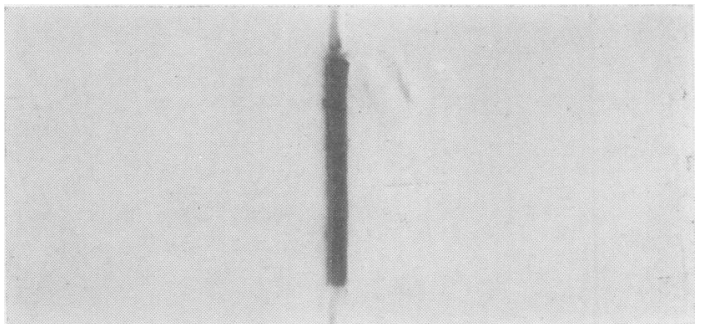

FIG. 3. Haemoglobin sample applied to block. Appearance before electrophoresis.

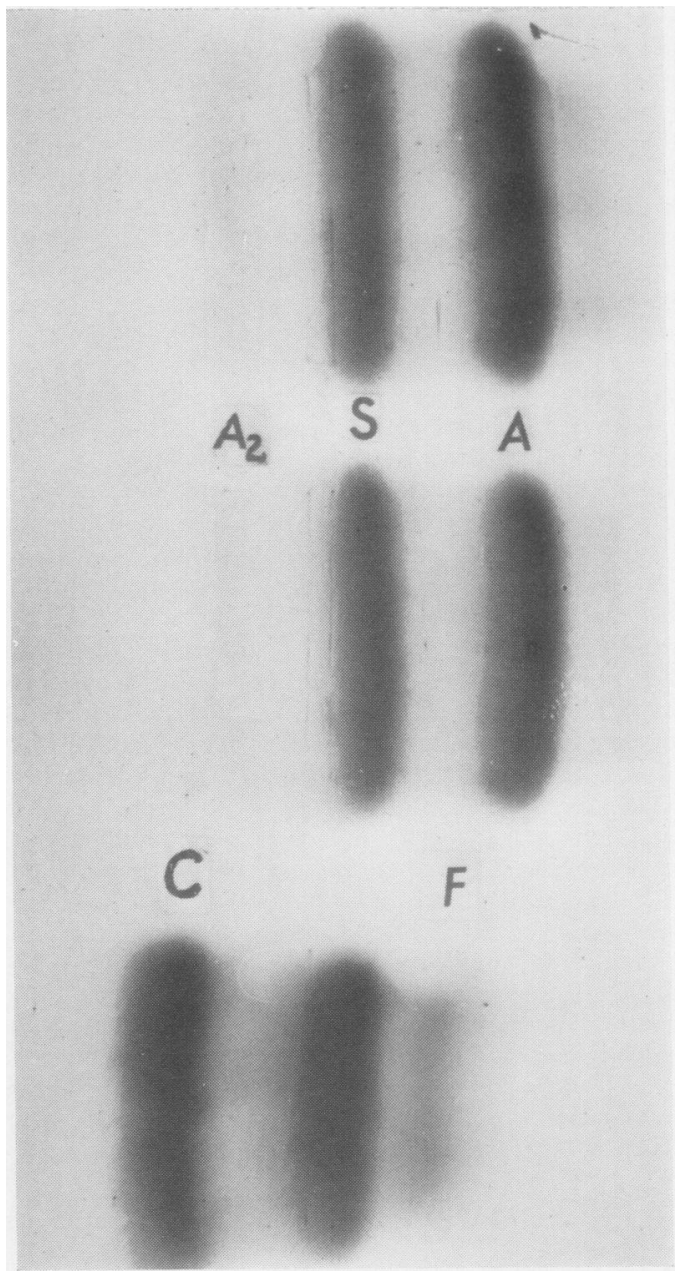

FIG. 4. Haemoglobin samples after electrophoresis, show-

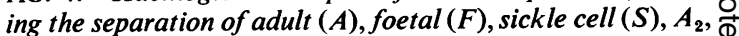
and $C$ haemoglobins. The two upper samples are from patients with sickle cell trait. The lower sample is from $a \stackrel{\Phi}{\Phi}$ patient with haemoglobin $S-C$ disease with increased amount of foetal haemoglobin. 
Houses), and when suitably dry, a ridge, $4 \times 0.3 \mathrm{~cm}$., is made on the block by means of a U-shaped piece of thin metal (Fig. 1). This 'ridger' was made from a piece of waste brass but any other thin metal should serve equally well. The open end of the 'ridger' is pressed firmly onto the block and pushed down evenly through the entire thickness $(0.5 \mathrm{~cm}$.) of the block. Three separate ridges are made on a block $20 \mathrm{~cm}$. wide (Fig. 2) and with a Pasteur pipette $0.2 \mathrm{ml}$. haemolysate is applied evenly to each ridge. In applying the haemolysate care must be taken to stop well short of the ends of the ridge; if this is not done the haemolysate will spread out beyond the confines of the ridge. After application of the haemolysate the gap between the ridge and the remainder of the block is closed by firm pressure with the fingers on both sides of the ridge. The appearance of the block at this stage is shown in Figure 3. Larger volumes of haemolysate (up to $1 \mathrm{ml}$.) can be applied by making a continuous ridge across the width of the block.

Electrophoresis is carried out in veronal buffer $(p \mathrm{H}$ 8.6 , ionic strength 0.05 ) for about 16 hours at $4^{\circ} \mathrm{C}$. with a current of 30 milliamps at 8 volts per centimetre length of block. The separation of some haemoglobins by this method is shown in Figure 4.

We are grateful to Professor P. L. Mollison for advice in the preparation of this communication and to the Photography Department, St. Mary's Hospital Medical School, for the photographs.

\section{REFERENCE}

Kunkel, H. G., and Wallenius, G. (1955). Science, 122, 288.

\section{A routine method for staining eosinophils in sputum}

\author{
RICHARD W. PAYNE and R. H. HILL From the \\ Department of Clinical Pathology, St. Albans \\ City Hospital, St. Albans, Herts.
}

The presence of eosinophils in the sputum of patients suffering from asthma or bronchitis accompanied by bronchospasm is often considered to be circumstantial evidence of an 'allergic' element in the aetiology of the bronchospasm. The interest of physicians in the presence or absence of eosinophils in the sputum of these patients has greatly increased since steroid treatment has been available. The following technique has been used for the past year and has been found to give clear-cut, reproducible appearances which are considerably easier to interpret than those seen in preparations stained with haematoxylin and eosin.

\section{METHOD}

Fix 'wet' smears of sputum overnight in methanol-ether at room temperature. Bring through alcohols to water. Stain in Ehrlich's haematoxylin for 15 minutes and 'blue' in tap water. If necessary differentiate in acid-alcohol ( $1 \%$ hydrochloric acid in spirit). 'Blue' in tap water. Counterstain in carbol-chromotrope $2 \mathrm{R}$ (see appendix) for 30 minutes. Wash in water. Dehydrate through the alcohols, clear in xylol, and mount in DPX.

\section{APPENDIX}

\section{CARBOL-CHROMOTROPE 2R}

$$
\begin{aligned}
& \text { Phenol crystals } \ldots \ldots \ldots 1 \cdot 0 \mathrm{~g} \text {. } \\
& \text { 'Chromotrope } 2 \mathrm{R} \ldots \ldots \ldots 0.5 \mathrm{~g} \text {. } \\
& \text { Distilled water } \ldots \ldots \ldots 100 \mathrm{ml} \text {. }
\end{aligned}
$$

Place the phenol crystals in a flask and warm under a hot tap. When crystals have melted add the Chromotrope $2 \mathrm{R}$ and the water.

${ }^{1}$ Obtainable from British Drug Houses Ltd., B.D.H. Laboratory Chemicals Division, Poole, Dorset.

Received for publication 11 January 1963. 